\title{
Is biology now part of physics?
}

\section{Reductionism is almost a dirty word, especially in biology, but after thirty years of DNA, it is high time that biologists paid attention to the question of what constitutes an explanation.}

IN the preface to the first edition of his great book on quantum mechanics, P.A.M. Dirac announced (in 1929) that physics and chemistry had become branches of applied mathematics. And although the sentence was significantly omitted when the second edition appeared in 1931, there is a sense in which Dirac's first claim was correct. For as events have shown, few parts of physical science are untouched by the microscopic view of reality for which the 1920s provided the capstone.

Naturally, classical mechanics still has practical uses, but even with seemingly classical problems, people are increasingly driven to microscopic considerations to check the plausibility of measured parameters. Moreover, it would be absurd to pretend that the most literal reading of Dirac's remark, that all macroscopic phenomena are accurately calculable from first principles and the parameters of the microscopic entities involved, will ever be valid.

The sense in which Dirac's remark has force is that there is reasonable confidence that the surviving impediments to the microscopic explanation of macroscopic phenomena are of two kinds only - computational difficulties and sheer ignorance. While computational difficulties are usually and easily forgiven, their obduracy does of course explain why there is no danger that moderate reductionism, the notion that the properties of wholes are functions of the properties of their parts which are themselves (give or take a quark or so) well known, will put macroscopic physical science out of business. That, in any case, would be assured by persisting ignorance, which appears to be of two kinds - ignorance of previously unknown phenomena (such as the shortage of neutrinos from the Sun) and ignorance born of failures of imagination (such as the compatibility of the physical phenomenon called chaos with the nonlinear deterministic equations that describe them). But with these qualifications, the weak reading of Dirac's claim makes sense and, indeed, has been a powerful stimulus of physical science in the past half-century.

So how soon will it be applicable to biology? At any time in the past thirty years, it might have been held that with the structure of DNA described (and confirmed by experiment), and the mechanisms of protein synthesis known, biology has also been made a branch of applied mathematics. But this is clearly far from being the case. For all the confidence with which nucleic acid polymers are described in high-school textbooks, even the simple physical chemistry of the double-stranded molecule leaves much unexplained. Empirically, quantities such as the enthalpy of hydrogen bonding between two strands can be worked out, but little is known about the corresponding interaction of single strands of DNA with water while almost next to nothing has been done to take account of the interactions between consecutive pairs of bases in a double-stranded molecule (which must nevertheless account for the differences of structure between lefthanded helices and the normal righthanded variety). More generally, and more worrying, the interaction of large molecules with the watery media in which they are usually found is not so much a puzzle as an issue tacitly recognized to be too complicated to tackle and best for the time being ignored.

In much the same way, the search for an explanation of how enzymes function is usually conducted on principles that fall short of full-blooded microscopic explanation. To be sure, there is now a long list of enzyme molecules whose interaction with the ligand molecule has been reasonably well inferred and whose catalytic effects can be explained by some supposed change of shape, an allosteric transformation. This, however, is a far cry from what might be thought proper if biology were already a part of physics - in principle, the prediction from the specification of some protein molecule of its function in real life. Moreover, it is plain that the difficulty cannot simply be written of as computational: the gap between what (elsewhere) can be calculated and the behaviour of apparently bistable molecules is too great to be spanned without a substantial knowledge empirically arrived at about the functioning of molecules that act as enzymes.

Exactly similar difficulties afflict other now familiar studies, the processing of DNA in cells for example. In bacteria, the characteristic places on DNA molecules at which transcription starts and, then, stops (and which thus define a gene) are known, but only in the sense that they have been recognized for what they are. While ignorance persists about the way in which the associated enzymes function, no would-be reductionist can write off the problem as a computational difficulty. And, as is well known, eukaryotic organisms present more serious problems. Who would pre- tend that given a sufficiently large computer, he could for example tell which parts of a stretch of DNA are functional and which are, outwardly at least, irrelevant?

The energetics of identifiable biochemical processes in cells points to a different kind of problem. The explanation of how cells can maintain large concentrations of materials such as ATP well away from what would be the natural condition of equilibrium, or transfer protons across cell membranes (as in oxidative phosphorylation) or sodium and potassium ions in different directions across neuronal membranes, can be described in terms consistent with thermodynamics. That is what nonequilibrium thermodynamics is for. But while the practitioners are confident enough when calculating (or saying that they could calculate with sufficient computing power) the voltage of a thermocouple (a simple system out of equilibrium), even the simplest processes within cells can be accounted for only by taking for granted the existence (again far from chemical equilibrium) of the complicated entities in which they occur.

This is why the reductionists are often opposed by those who argue that while their programme may work in physics, it is bound to be defeated in biology not merely by the lack of credibly sufficient computing power but by the sheer complexity of living systems. Complexity makes a qualitative difference - and, in any case, the search for microscopic explanations of macroscopic happenings is, in this case, a poor guide to the design of fresh experiments and observations.

But is that necessarily the case? In physical science, the search for microscopic explanations has been remarkably productive in the past halfcentury, especially because people have sensibly not followed the bigoted course of pretending that only microscopic explanations matter. The truth is that microscopic explanations are in principle simpler, and often more stimulating as well, than those which are essentially correlations between one set of phenomena (effects) and another ("causes"). Molecular biologists might prudently keep those benefits more clearly in mind than seems customary in the present rush to compile what might be called the natural history of the gene, if only because the only possible answer to the question at the top of this page is "not yet".

John Maddox 